\title{
Cystic fibrosis-related diabetes: links, challenges, and future directions
}

This article was published in the following Dove Press journal:

Research and Reports in Endocrine Disorders

5 November 2015

Number of times this article has been viewed

\author{
Saba Sheikh \\ Andrea Kelly \\ Department of Pediatrics, The \\ Children's Hospital of Philadelphia, \\ Philadelphia, PA, USA
}

Correspondence: Saba Sheikh

The Children's Hospital of Philadelphia,

350I Civic Center Blvd, Philadelphia,

PA 19104, USA

$\mathrm{Tel}+$ I 2155903749

Fax + I 2155903500

Emailsheikhs@email.chop.edu

\begin{abstract}
Cystic fibrosis-related diabetes (CFRD) is a common comorbidity in cystic fibrosis (CF) and portends worse clinical outcomes including lower lung function and nutritional status, and decreased survival. CFRD is distinct from other forms of diabetes, but relative insulin deficiency is a predominant feature. The catabolic effects of insulin deficiency coupled with the pro-inflammatory effects of hyperglycemia likely affect clinical outcomes. Posited mechanisms of CFRD development include collateral damage from pancreatic exocrine destruction, inherent $\beta$-cell defect, CFTR dysfunction, and incretin deficiency or unresponsiveness. Even though CF clinical care teams are increasingly aware of its implications, CFRD screening and management remain a challenge. Promising clinical and basic research in the fields of diabetes and CF and the advent of novel therapeutics targeting the protein defect in CF have potential to change CFRD care. This review presents newer data on insulin defects and glucose derangements and highlights continuing challenges and unanswered questions.
\end{abstract}

Keywords: cystic fibrosis, insulin, glucose intolerance

\section{Background}

With medical advances in the field of cystic fibrosis (CF), individuals with $\mathrm{CF}$ are now surviving into adulthood. This improved survival is accompanied by an increasing prevalence of non-pulmonary comorbidities that have the potential to threaten pulmonary health. Affecting $40 \%-50 \%$ of adults age $>30$ years, cystic fibrosis-related diabetes (CFRD) is particularly problematic. ${ }^{1}$ It is associated with worse pulmonary function, greater nutritional decline, and increased mortality. ${ }^{2}$ The relationship between CFRD and adverse clinical outcomes highlights the importance of an improved understanding of insulin deficiency and hyperglycemia in CF, with the ultimate goals of either slowing the progression or altogether preventing diabetes development and mitigating the negative effects in the setting of established diabetes. CF caregivers now recognize the importance of CFRD and great strides have been made in developing an understanding of the disease but many questions with respect to pathophysiology, screening including frequency and timing, and even nature of intervention remain unanswered.

\section{Clinical significance}

Since the 1980s, the CF community has recognized that diabetes portends worse survival in $\mathrm{CF}^{3}$ Only $25 \%$ of individuals with CFRD survived to age 30 years compared to the $60 \%$ without CFRD. ${ }^{3}$ What remained unresolved, however, was the extent to which diabetes directly contributed to premature death versus (vs) represented a surrogate for worse CF disease. Indeed, CFTR mutation severity impacts not only the risk of 
diabetes development but mortality as well. ${ }^{4}$ Subsequently, early identification and treatment with insulin were associated with improved survival in CFRD. ${ }^{2}$ Despite this improvement, mortality remains over three times higher in patients with CFRD than in individuals without CFRD (1.8 vs 0.5 per 100 person-years). ${ }^{4}$ Moreover, although CFRD prevalence is higher at every age in females than in males, CFRD has been associated with worse survival to a greater degree in males than females. ${ }^{4}$ The mechanism underlying this sex difference is unknown. Recent work identifying greater lean body mass deficits in males than females with CF may represent one avenue by which CFRD differentially affects CF outcomes. ${ }^{5}$

CFRD is identified as a preventable cause of the decline in forced expiratory volume in 1 second $\left(\mathrm{FEV}_{1}\right)$, a marker of pulmonary function. ${ }^{6}$ Lower percent predicted $\mathrm{FEV}_{1}$ (FEV ${ }_{1}$ \%-predicted) is seen in all age groups with CFRD. ${ }^{7}$ The rate of decline in $\mathrm{FEV}_{1}$ is higher in CFRD compared to $\mathrm{CF}$ patients with normal glucose tolerance (NGT), ${ }^{8}$ can begin several years before CFRD is diagnosed, ${ }^{3,9}$ and is correlated with degree of insulin deficiency. ${ }^{8}$ Time to next exacerbation is a health outcome often used in clinical trials in CF, and CFRD is associated with shortened duration between CF exacerbations, although older age may be an important confounder in this relationship. ${ }^{10}$ Not surprisingly, CFRD is also associated with earlier age at lung transplantation. ${ }^{11}$ Post-transplant diabetes is common in CF, but the impact of pre-transplant CFRD upon graft survival is not well defined. ${ }^{12}$ The mechanisms linking CFRD with inflammation and pulmonary decline have yet to be resolved. In vitro studies identifying increased growth of Staphylococcus aureus and Pseudomonas aeruginosa with increasing airway surface liquid glucose concentration ${ }^{13,14}$ support a direct role for hyperglycemia promoting pathogens. However, sputum glucose values are not elevated in CFRD patients. ${ }^{15}$ This lower glucose may represent increased substrate uptake by a higher concentration of airway bacteria; alternatively, mechanisms other than the pro-infectious nature of chronic hyperglycemia may be operative. Treatment of CFRD at least partially reverses the pulmonary decline experienced by $\mathrm{CF}$ patients, ${ }^{16-18}$ but the direct pulmonary effects of insulin treatment vs lower glucose have yet to be resolved.

Since insulin is an anabolic hormone, insulin deficiency in CFRD can worsen nutritional status. Lower body mass index (BMI) has been observed in CFRD. ${ }^{7}$ CFRD at a young age reveals trends in shorter adult height; ${ }^{19,20}$ target adult height is not achieved despite treatment with insulin. ${ }^{20}$ Lower BMI can be observed before the CFRD diagnosis is made, ${ }^{16,21}$ but early insulin treatment has not consistently been associated with improved nutritional status in the setting of pre-diabetes. ${ }^{22-24}$ The inconsistent findings may be related to suboptimal insulin dosing and are likely hampered by the injections required for insulin administration.

Aggressive diabetes treatment in the non-CF population is routed in preventing microvascular (and macrovascular) complications, but the shortened life expectancy in CF previously obviated these concerns. These complications, however, are a reality in $\mathrm{CF}$ especially in the presence of fasting hyperglycemia $(\mathrm{FH}) .{ }^{25}$ In individuals with $\mathrm{CFRD}$ with $\mathrm{FH} \geq 10$ years, $14 \%$ had microalbuminuria and $16 \%$ retinopathy. In a separate study in which presence of FH was not clearly defined, subjects with CFRD were more likely than subjects with type 1 diabetes mellitus (T1DM) to have microalbuminuria, whereas retinopathy was less common. ${ }^{26}$ People with $\mathrm{CF}$ already have multiple risk factors for renal dysfunction, including chronic inflammation and frequent usage of nephrotoxic drugs, and CFRD can compound this risk. Individuals with CFRD for 1-4 years vs CF individuals without CFRD were greater than two times more likely to have chronic kidney disease (hazard ratio $2.40,95 \%$ confidence interval $1.74-3.32$ ), and those who had disease $>5$ years were more than five times more likely to have chronic kidney disease (hazard ratio $4.56,95 \%$ confidence interval 2.84-7.31). ${ }^{27}$ The impact of aggressive CFRD treatment upon these outcomes has yet to be elucidated.

\section{Pathophysiology}

The American Diabetes Association classifies diabetes based on etiology, with CFRD categorized under "other form of diabetes" or pancreatogenic diabetes. ${ }^{28}$ The primary defect in $\mathrm{CF}$ is that of insulin deficiency. The prevailing hypothesis for insulin deficiency in $\mathrm{CF}$ is that exocrine pancreatic damage incurred from viscous secretions spills over to damage the endocrine pancreas. As such, islet function is affected globally and not limited to $\beta$-cells. ${ }^{29-31}$ In support of this hypothesis, CFRD occurs more commonly in the presence of the severe $\mathrm{CF}$ mutations associated with pancreatic exocrine insufficiency. ${ }^{32}$ However, while autopsy studies of CF pancreas do reveal loss of $\beta$-cell mass, the extent of $\beta$-cell mass loss does not completely agree with presence or absence of diabetes suggesting additional mechanisms are at play.

This insulin deficiency largely manifests as post-prandial hyperglycemia due to delayed early insulin secretion, ${ }^{33}$ but basal insulin secretion is largely preserved until late in the course of CFRD. In non-diabetic CF individuals, despite NGT, $\beta$-cell secretory capacity is reduced. ${ }^{34}$ In response to oral or intravenous glucose challenge, $\mathrm{CF}$ individuals with 
NGT have delayed and blunted insulin secretion compared to controls, ${ }^{35}$ and further differences can be elicited in these individuals based on pancreatic insufficiency status; whereas NGT-CF individuals who are pancreatic sufficient show normal insulin secretion, it is delayed and blunted in those who are pancreatic insufficient. ${ }^{36}$ As glucose tolerance worsens, time to peak insulin increases and peak values decrease. ${ }^{36,37}$ During intravenous glucose tolerance testing and hyperglycemic clamp, acute insulin responses in subjects with CFRD were found to be dampened compared to controls. ${ }^{38}$ Serial oral glucose tolerance tests (OGTTs) performed on CF individuals demonstrate that over time insulinopenia and glucose intolerance worsens. ${ }^{39,40}$ Incretin hormones are released from neuroendocrine cells in the gut and augment insulin secretion in response to nutrients. Defects in their secretion have been reported in chronic pancreatitis. ${ }^{41}$ and may also be operative in pancreatic insufficient CF. Studies of incretins, GIP, and GLP-1, have reported a spectrum of results, from normal basal secretion, to elevation in response to an oral glucose challenge to no change with a glucose challenge. ${ }^{42}$ Dietary constituents, including fatty acids, modulate insulin secretion, and, thus, a mixed meal tolerance test is likely a more physiologic test. Indeed, pancreatic enzyme replacement appears to improve GIP secretion and glucose excursion in individuals with pancreatic insufficient $\mathrm{CF}^{43}$ The role of incretin-based therapy in $\mathrm{CF}$ is the subject of active investigation (principal investigator: Stecenko - https:/clinicaltrials.gov/ct2/show/ NCT00967798; Kelly/Rickels - NCT01879228).

Insulin resistance is not considered a pronounced feature of CF. ${ }^{33,44}$ Studies using oral and intravenous glucose challenges have reported normal insulin sensitivity, which does not worsen over time. ${ }^{39,40,45}$ There is emerging evidence for insulin resistance in $\mathrm{CF}$; a decrease in gluconeogenesis due to insulin signal transduction defect caused by reduction of the transcription factor, FOXO1, was recently discovered by Smerieri et al. ${ }^{46}$ Insulin resistance emerges during periods of stress and is evident in the impaired insulin-mediated suppression of proteolysis in $\mathrm{CF}^{47,48}$ The degree of suppression of proteolysis is linked to the degree of glucose intolerance with worsening glucose tolerance correlating with greater impairments in suppression of proteolysis. ${ }^{48}$ Obesity is now reported in $\mathrm{CF}$ patients and may confer an insulin resistant state to confound underlying insulin secretion defects in $\mathrm{CF}$; the contribution of obesity to CFRD development will be an important consideration.

Amyloid deposition features in both type 2 diabetes mellitus (T2DM) and CF and, while not well understood, may be a marker of endoplasmic reticulum (ER) stress. Normal ER function is essential for protein folding in all eukaryotic cells; misfolding triggers an adaptive response, failure of which results in $\beta$-cell apoptosis. Post-mortem studies in T2DM identify decreased $\beta$-cell mass and markers of apoptosis. ${ }^{50,51}$ Excessive insulin demand imposed by insulin resistance, obesity-related inflammation - so called "metainflammation", ${ }^{52}$ lipotoxicity arising from excess free fatty acids, and hypoxia are all thought to give rise to ER stress that contributes to T2DM. Their role in CF has not been delineated but given that individuals with worse $\mathrm{CF}$ lung disease are more likely to have CFRD, it is reasonable to hypothesize that the inflammation of CF lung disease may also contribute directly to $\beta$-cell dysfunction and insulin deficiency. Also leading to inflammation and insulin resistance in CF may be the presence of impaired autophagy, a key process in clearing misfolded proteins which is hypothesized to be deficient in CF epithelial cells. ${ }^{53}$ Autophagy may avert $\beta$-cell dysfunction and apoptosis in T2DM, and its loss in murine models of T2DM can lead to insulin resistance and inflammation. ${ }^{54}$

Additional studies find similarities between CFRD and T2DM. Twin studies suggest that the risk of CFRD closely correlates with certain genes associated with T2DM, such as TCF7L2. ${ }^{55}$ Genome-wide association studies have identified four more novel genetic modifiers of CFRD: SLC26A9, $C D K A L 1, C D K N 2 A / B$, and $I G F 2 B P 2$, all of which are known susceptibility alleles for T2DM. ${ }^{56}$ Given the variable development of CFRD in CF patients with DF508 and genes associated with T2DM, epigenetic factors may play a yet unknown role in the pathogenesis of CFRD. Histone modification and DNA methylation causing altered gene expression and impaired insulin secretion in animal studies suggest presence of epigenetic factors in T2DM development. ${ }^{57,58}$ Differential DNA methylation of CpG sites within TCF7L2 gene loci and its effect on insulin secretion in T2DM, ${ }^{59}$ may be of interest in CFRD as well.

Finally, the direct role of defective CFTR function in $\mathrm{CF}$ is not known. Animal models are providing important insights. In rats, CFTR is expressed in pancreatic islet $\alpha$-cells and to a lesser extent $\beta$-cells. ${ }^{60}$ The CF ferret model reveals early dysregulated insulin secretion that appears to pre-date extensive pancreatic exocrine damage.$^{61}$ Glucose stimulated insulin secretion is attenuated in CFTR mutant (DF508) mice compared with wild-type mice, and VX-809 a corrector of DF508 mutation helps reverse this dampened response. ${ }^{62}$ The CF pig model similarly reveals glucose and insulin secretion abnormalities in the absence of loss of islet mass. ${ }^{63}$ Together these data support a direct role of CFTR in pancreatic islet function. Reports of improved glucose status in patients 
with at least one of ten rare mutations, who are treated with the CFTR modulator, ivacaftor, ${ }^{64,65}$ are prompting human investigations of the underlying mechanisms.

\section{Screening and diagnosis Glucose tolerance tests}

The onset of glucose abnormalities in CF is insidious and glucose derangements should be suspected with declining clinical status not amenable to direct pulmonary care and improved nutrition. The Cystic Fibrosis Foundation (CFF) and International Society of Pediatric and Adolescent Diabetes have recommended annual screening with an OGTT $(1.75 \mathrm{~g} / \mathrm{kg}$ glucose; maximum dose $75 \mathrm{~g}$ ) starting by age 10 years. ${ }^{66}$ Glucose tolerance fluctuates over a wide spectrum and can be characterized by an OGTT. Four categories of glucose tolerance are currently defined by the OGTT: NGT, indeterminate, impaired glucose tolerance (IGT) and CFRD, Table 1. CFRD with and without FH may be distinguished to communicate degree of insulin deficiency and microvascular disease risk, but both are treated based upon their negative impact upon nutritional status. ${ }^{67} \mathrm{CFF}$ guidelines have adopted the same cutoffs for diagnosis as those used for T2DM. However, glucose thresholds for defining T2DM have been developed largely based on the risk of microvascular complications. In $\mathrm{CF}$, since pulmonary complications prevail, the relevance of lower thresholds for pulmonary, nutritional, and progression to diabetes risk are important considerations.

Variability in OGTT results is also of concern; the coefficient of variation for 2-hour blood glucose was as high as

Table I Screening and diagnosis of CFRD

\begin{tabular}{llll}
\hline OGTT & Annually $\geq 10$ years & & \\
\cline { 2 - 4 } & $\begin{array}{l}\text { Glucose tolerance } \\
\text { category }\end{array}$ & I-hour PG & 2-hour PG \\
& Normal (NGT) & $<140 \mathrm{mg} / \mathrm{dL}$ & $<140 \mathrm{mg} / \mathrm{dL}$ \\
Impaired (IGT) & $\mathrm{NA}$ & $>140 \mathrm{and}$ \\
& & $<200 \mathrm{mg} / \mathrm{dL}$ \\
& Indeterminate (INDET) & $>200 \mathrm{mg} / \mathrm{dL}$ & $<140 \mathrm{mg} / \mathrm{dL}$ \\
CFRD & $\mathrm{NA}$ & $>200 \mathrm{mg} / \mathrm{dL}$
\end{tabular}

HbA $_{\mathrm{lc}} \quad \geq 6.5 \%$ for diagnosis

$<6.5 \%$ does not exclude diagnosis

Fasting and During hospitalizations or intercurrent illnesses,

2-hour post- systemic glucocorticoid use monthly during and

prandial glucose after continuous overnight feeds

Random glucose CFRD $\geq 200 \mathrm{mg} / \mathrm{dL}+$ polyuria and polydipsia

Abbreviations: CFRD, cystic fibrosis-related diabetes; $\mathrm{HbA}_{t c}$, hemoglobin; $\mathrm{A}_{t c}$; IGT, impaired glucose tolerance; INDET, indeterminate glucose tolerance; NA, not applicable; NGT, normal glucose tolerance; OGTT, oral glucose tolerance test; PG, plasma glucose.
$25.3 \%$ in a German study of 1,128 subjects who underwent two OGTTs. ${ }^{68}$ Annual screening in CF patients also identifies large variability over time, and abnormal OGTT results can revert in the subsequent year. ${ }^{69,70}$ Additionally mid-OGTT glucose values at 30, 60, and 90 minutes and the glucose area under the curve for OGTT differ significantly between non-CF controls and CF patients with NGT. ${ }^{71}$ Moreover, clinicians frequently observe a discrepancy between OGTT and post-prandial glucose: 2-hour post-prandial glucose may be relatively normal in the context of eating mixed meals, Figure 1. The best approach to address this apparent insulin deficiency is not clear.

The presence of DF508 mutation is probably the most important factor influencing OGTT results. ${ }^{72,73}$ Homozygosity of DF508 is closely linked to abnormalities in glucose tolerance seen on OGTT. While the clinical severity of CF disease assessed using Shwachman-Kulczycki scores did not appear to be linked to this observation, ${ }^{72,73}$ CFRD is associated with worse pulmonary status. ${ }^{74}$ and severe $\mathrm{CF}$ mutations may increase pancreatic damage. Factors such as sex and anthropometric features have less of an impact on glucose tolerance. ${ }^{75}$

Particularly problematic, adherence to screening recommendations is generally poor, ${ }^{76}$ and from clinical experience, the OGTT is an unpopular test - it involves fasting and two to three blood draws. Lee et $\mathrm{al}^{77}$ tested the non-fasting 1-hour 50 g glucose challenge test in adults and suggested a plasma glucose $>140 \mathrm{mg} / \mathrm{dL}(7.8 \mathrm{mmol} / \mathrm{L})$ could identify individuals at increased risk for glucose abnormalities. A CFF-funded multicenter trial assessing the glucose challenge test is ongoing.

\section{Continuous glucose monitoring (CGM)}

With these limitations of an OGTT, the utility of CGM in CF has been the subject of investigation. CGM, which is validated in diabetic populations, ${ }^{78}$ measures interstitial glucose that is then translated into blood glucose. CGM has identified higher peak glucose levels in NGT CF people than healthy controls. ${ }^{71}$ Franzese et $\mathrm{al}^{79}$ observed discordance between OGTT and CGM results; the OGTT underestimated the degree of glucose intolerance. Figure 1 highlights the discordance in OGTT and CGM results in an adult $\mathrm{CF}$ female. In one study of $22 \mathrm{CF}$ subjects with NGT, a peak CGM glucose level of $182 \mathrm{mg} / \mathrm{dL}$ was identified, and observed post-prandial glucose peaks $>200$ $\mathrm{mg} / \mathrm{dL}$ in one-third of CF subjects with NGT, half of IGT subjects, and all patients with CFRD.${ }^{80}$ Glucose excursions $>200$ $\mathrm{mg} / \mathrm{dL}$ on CGM successfully predicted the development of IGT or CFRD as diagnosed by OGTT in a 2.5-year period. ${ }^{81}$ Hameed 


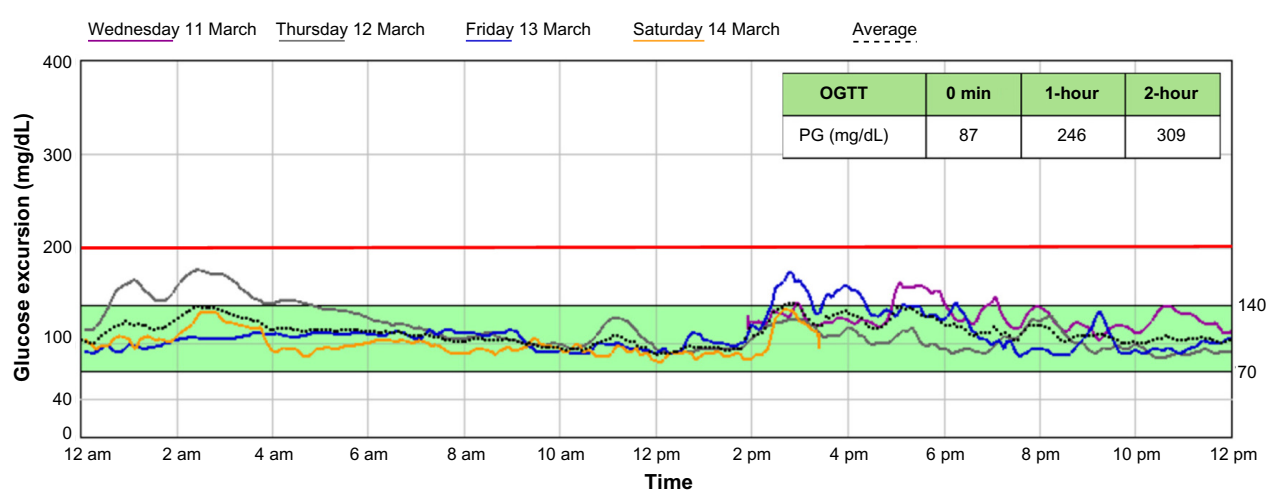

Figure I Patient $\mathrm{I}$ is an 18 years and 4 months old female who underwent CGM and OGTT testing during a 2 week period. Note: Glucose excursions on CGM are milder compared to those seen on OGTT.

Abbreviations: CGM, continuous glucose monitoring; min, minute; OGTT, oral glucose tolerance test; PG, plasma glucose.

et $\mathrm{al}^{21}$ found glucose $>140 \mathrm{mg} / \mathrm{dL}$ during CGM was correlated with greater declines in weight- $Z$ and pulmonary function over the previous year in children defined as having NGT or IGT by OGTT. O'Riordan et al ${ }^{82}$ confirmed the validity and reproducibility of CGM in over 100 children with CF.

The major disadvantage of performing CGM is placement of a subcutaneous probe that monitors glucose over 3-5 days; blood glucose by finger stick must also be performed multiple times per day for calibration of the CGM. CGM is presently not recommended as a diagnostic tool for CFRD by the US CFF. Instead, it is considered a complementary tool in the management of CFRD. ${ }^{66}$ The glucose excursions on CGM while not diagnostic of CFRD may be important in individual patients in whom clinical decline may be suspected and in whom early insulin treatment can improve clinical status. ${ }^{18}$

\section{Glycosylated hemoglobin}

Hemoglobin $\mathrm{A}_{1 \mathrm{c}}\left(\mathrm{HbA}_{1 \mathrm{c}}\right)$ closely correlates with mean plasma glucose in T1DM but a similar correlation does not seem to exist for $\mathrm{CF}^{83}$ Despite higher mean $\mathrm{HbA}_{1 \mathrm{c}}$ values in CF patients with diabetes than in those with NGT, ${ }^{11}$ a large proportion of diabetic $\mathrm{CF}$ individuals have normal $\mathrm{HbA}_{1 \mathrm{c}}{ }^{11,70,84}$ Thus, the CFF does not recommend use of $\mathrm{HbA}_{1 \mathrm{c}}$ for diabetes screening due to its poor sensitivity in the CF population. ${ }^{66}$ The utility of a lower $\mathrm{HbA}_{1 \mathrm{c}}$ threshold to identify the subset of patients at greatest risk for CFRD and who, therefore, should undergo an OGTT has recently been addressed by Burgess et al. ${ }^{85}$ Their study identified a threshold of $\geq 5.8 \%$ as compared to $\geq 6.5$ for T1DM and T2DM as sufficiently sensitive to screen for CFRD and to identify individuals whose "elevated" $\mathrm{HbA}_{1 \mathrm{c}}$ should be followed by a diagnostic OGTT.

\section{Random blood glucose monitoring}

Using only random blood glucose for the diagnosis of CFRD is challenging. An isolated elevated glucose level $(>200 \mathrm{mg} / \mathrm{dL})$ occurring within 2 hours post-meal (as evident by CGM) suggests an insulin defect but does not translate into diabetes. Random blood glucose checks - fasting and 2-hour postprandial, are recommended for CF patients within the first 48 hours of an acute illness requiring intravenous antibiotics or glucocorticoids based on expert consensus. ${ }^{66}$ These elevations require confirmation by serum glucose measurements but can identify individuals who are at risk for diabetes. In the outpatient setting, glucose should be measured periodically during and after continuous overnight gastrostomy tube (G-tube) feeds. ${ }^{66}$ Figure 2 shows two CF subjects with NGT on OGTT followed at our $\mathrm{CF}$ center who experienced worsening BMI despite optimum nutrition and pulmonary care but who demonstrated hyperglycemia associated with G-tube feeds. On initiation of insulin, the nutritional status of both patients improved. Since G-tube feeds are routinely instituted in CF patients with low BMI, of particular interest in this population is whether continuous overnight feeds "overburden" the pancreas.

\section{Significance of early glucose abnormalities}

While the significance of CFRD and the treatment recommendations are relatively clear, early glucose abnormalities pose a challenge. During this "pre-diabetic" state, clinical decline has already ensued. ${ }^{9,18}$ This pre-diabetic state is based on conventional OGTT criteria and may be the byproduct of nonCF specific diagnostic cutoffs. The lag in CFRD diagnosis from clinical decline could suggest an inappropriate delay in treatment as clinical decline has already started. Given this consideration, some investigators have focused on the impact 

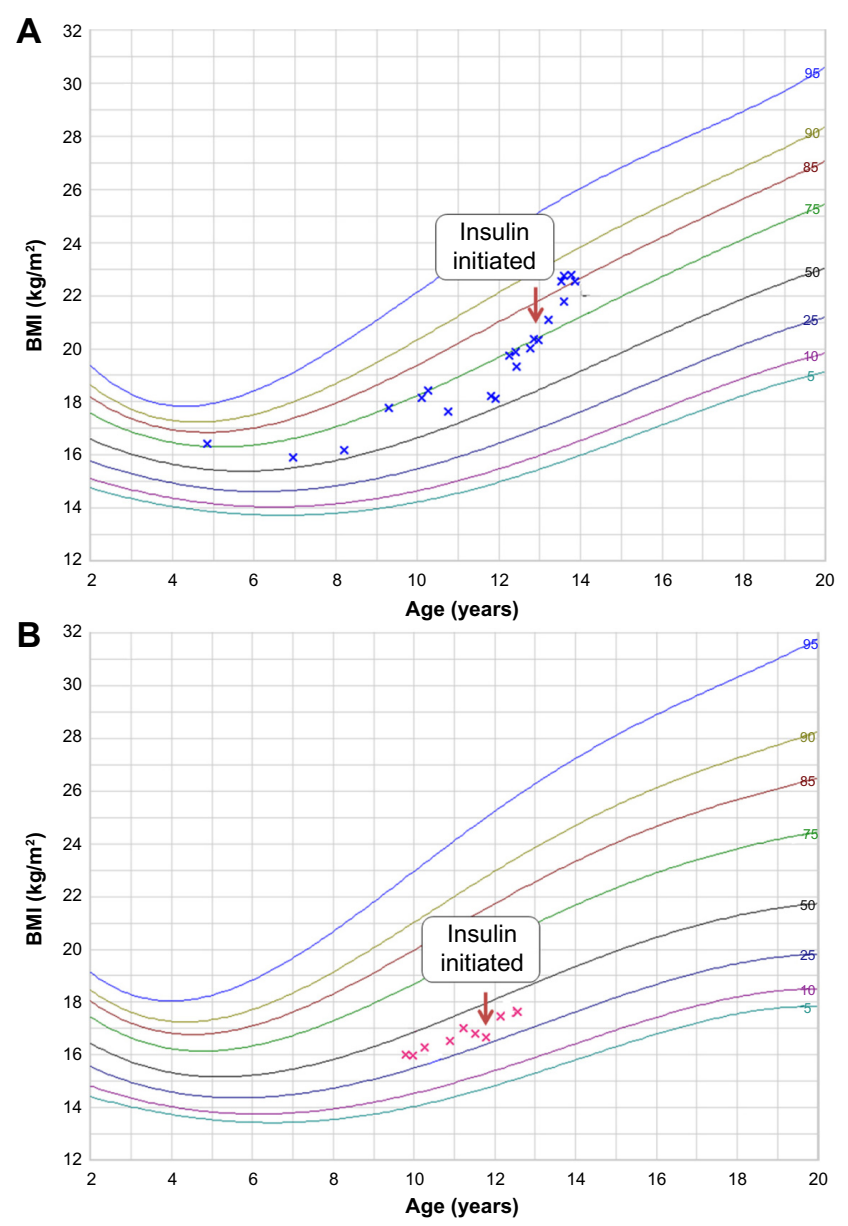

Figure 2 Improvements in BMI on insulin treatment among two individuals with normal OGTT but elevated random post-prandial blood glucose (BG) monitoring. Notes: Patient I (A) is a male, 13 years and 4 months old with pancreatic insufficient $\mathrm{CF}$ on long-term G-tube supplementation who showed BG results in the mid-300s mg/dL on overnight feeds. Patient 2 (B) is a female, 11 years and 6 months old who demonstrated BG $>200 \mathrm{mg} / \mathrm{dL}$ immediately during overnight feeds.

Abbreviations: BMI, body mass index; OGTT, oral glucose tolerance test; CF, cystic fibrosis; G-tube, gastrostomy tube.

of pre-CFRD glucose abnormalities on clinical outcomes and early treatment to prevent the associated decline.

Recent evidence suggests mild glucose derangements are not benign and are associated with clinical deterioration pulmonary function and nutritional status worsen in pre-diabetes. $^{3,9}$ The CFF has recognized that IGT may be important, but its consequences are unclear and therefore no specific guidelines for management have been recommended. ${ }^{86}$ Tofe et $\mathrm{al}^{87}$ evaluated lung function and nutrition in patients with IGT and demonstrated significantly lower FEV 1 and BMI values compared to CF patients with NGT. Diminished insulin secretion and increased insulin resistance were present in patients with IGT compared to those with NGT. ${ }^{87}$ Therefore, insulin treatment may be beneficial in the pre-diabetic state, and a few small studies lend support to this approach. ${ }^{22}$ Bizzarri et $\mathrm{al}^{22}$ reported improvements in $\mathrm{BMI}$ and $\mathrm{FEV}_{1} \%$ with glargine treatment in a small population of CF patients with IGT. Moran et $\mathrm{l}^{67}$ treated 20 patients with IGT with rapid acting insulin three times per day with meals and found no improvements in BMI. Minicucci et al ${ }^{88}$ treated 16 patients with glargine and did not demonstrate improvements in BMI and $\mathrm{FEV}_{1} \%$. These studies are small, with a high proportion of dropouts, and also differed in the insulin dosages used. CFIDEA, a larger randomized controlled trial using once-daily insulin determined to evaluate the benefits of treatment of early insulin deficiency is currently in progress (clinicaltrials. gov identifier: NCT01100892).

\section{Treatment}

The treatment of choice in CFRD is insulin; insulin administration has an established benefit for CF patients. Nutritional outcomes in patients diagnosed with CFRD improve with the initiation of insulin. ${ }^{16,67,89}$ as does pulmonary function. ${ }^{16,23}$ The decline in $\mathrm{BMI}$ and $\mathrm{FEV}_{1}$ \%-predicted that is present in CFRD patients pre-treatment and likely also in the pre-diabetic phase is reversed with insulin. ${ }^{16,89,90}$ Insulin treatment may also decrease the frequency of pulmonary exacerbations. ${ }^{24}$

However, CFRD treatment is an additional burden on patients with $\mathrm{CF}$, who are already on complex medical regimens for maintenance of pulmonary and nutritional health. Management of CFRD with insulin can be demanding, requiring frequent blood glucose checks, subcutaneous injections, and carbohydrate counting.

Oral hypoglycemic agents are not recommended for treatment of CFRD as data on efficacy are limited. A recent Cochrane review noted that there is no established advantage of oral hypoglycemics over insulin and further studies are needed to establish benefit. ${ }^{91}$ A single trial was identified where 12-month treatment with repaglinide, an oral insulin secretagogue, did not improve $\mathrm{BMI}$ or $\mathrm{FEV}_{1}$. The side effects of insulin sensitizers too may be problematic in CF individuals. Metformin favors weight loss in diabetes, can cause metabolic acidosis and gastrointestinal symptoms, and is contraindicated in renal impairment whereas thiazolidinedione causes osteoporosis. Based on their case report of a 48-year old female with CFRD who benefited from islet cell transplantation, Spijker et $\mathrm{al}^{92}$ have recommended islet transplantation after lung transplantation as an option for CFRD treatment in certain patients.

Novel therapeutic options include medications that target the incretin system. Incretin hormones, GLP-1 and GIP induce insulin release from the pancreas but have a short half-life as they are inactivated by enzyme DPP4. DPP4 inhibitors such as sitagliptin are a new class of 
anti-hyperglycemic drugs that reduce degradation of incretins, enhance insulin secretion, and limit post-prandial hyperglycemia. GLP-1 agonists used in the treatment of T2DM may help avoid the hypoglycemic effects of insulin therapy.${ }^{93}$ These agents also induce weight loss, ${ }^{94}$ which can be a potential drawback in CF subjects. However, with the reported increase in obesity among CF subjects, ${ }^{49}$ GLP-1 agonists and DPP4 inhibitors may have an added role in the obese sub-group of CF subjects. Concern has arisen regarding GLP-1 analogs and cases of drug-induced pancreatitis, but the extent to which these analogs cause pancreatitis is still debated. Until more data are available, incretin-based therapies should be avoided in pancreatogenic diabetes. ${ }^{95}$ In pancreatic insufficient $\mathrm{CF}$, pancreatitis is not expected. Other agents yet to be explored in CFRD include SGLT2 inhibitors and IGF-I. There is limited experience using SGLT2 inhibitors in T2DM but they are promising agents. ${ }^{96}$ IGF-I, which complements insulin action, has been used for treatment in combination with insulin. ${ }^{97}$ IGF-I treatment in vitro and in vivo in $\mathrm{CF}$ mouse skeletal muscles improves insulin signaling. ${ }^{46}$

CF-causing mutations may lead to: 1) defective production of CFTR, 2) failure of the CFTR to be presented to and function at the plasma membrane or 3) defective CFTR conductance - ie, the probability of the chloride channel being open to transport chloride is decreased. Drugs that improve CFTR production and transit to the apical membrane, so-called correctors, and those that improve conductance, referred to as potentiators, may modulate pancreatic $\beta$-cell function as well. CFTR potentiator, ivacaftor, is available for a small subset of CF patients, those with one of ten rare mutations, and the treatment of this subset's defective CFTR conductance with ivacaftor results in clinically significant improvements in pulmonary function, weight, and BMI. ${ }^{98}$ Interestingly, improvements in insulin secretion during OGTT and acute glucose infusion were found in a subset of five patients following ivacaftor treatment,${ }^{64}$ leading the authors to speculate whether initiation of a potentiator prior to $\beta$-cell failure can delay or prevent CFRD. Hayes et $a l^{65}$ reported a case in which ivacaftor treatment resulted in resolution of diabetes, suggesting that ivacaftor may in fact also treat CFRD. Figure 3 shows improvements in BMI and normalization of OGTT after initiation of ivacaftor in a 13-year-old female with G551D mutation and CFRD without FH. The impact of these CF-specific therapies may extend beyond pulmonary function and nutrition and further investigation on the impact

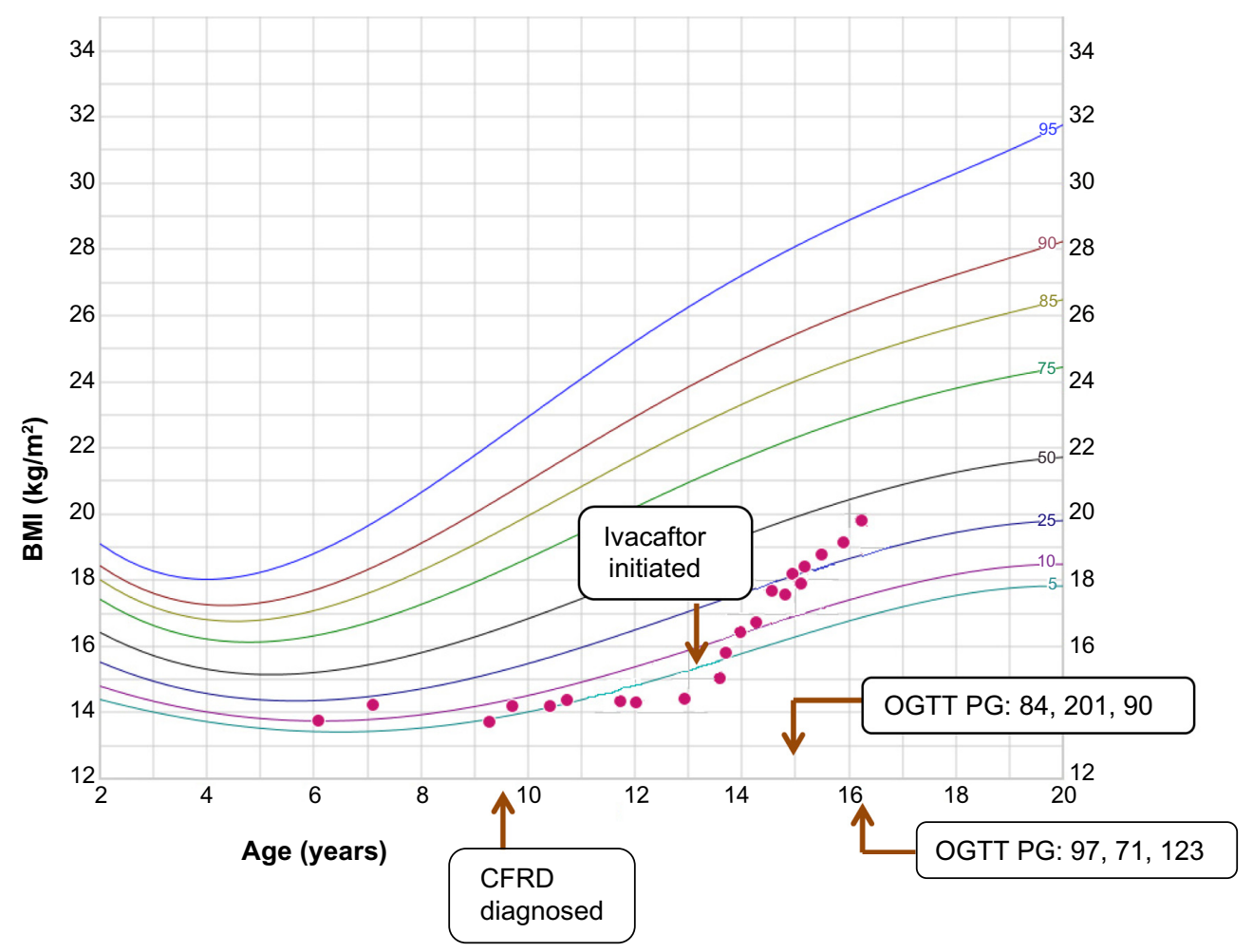

Figure 3 Patient with one G55ID mutation, diagnosed with CFRD at 9 years and 5 months who initiated ivacaftor at I3 years 4 months old.

Note: The patient's BMI and BG on home glucose monitoring improved and CFRD treatment was stopped; the individual subsequently has remained off CFRD treatment with improvements in OGTT plasma glucose (PG; fasting, I- and 2-hours).

Abbreviations: CFRD, cystic fibrosis-related diabetes; BMI, body mass index; BG, blood glucose; OGTT, oral glucose tolerance test. 
of ivacaftor upon insulin secretion and glucose tolerance in CF patients with G551D mutation is underway (clinicaltrials. gov identifier: NCT02039986).

\section{Quality of life}

The diagnosis of diabetes places an additional burden on patients already managing the complexities of CF care. Adding to the difficulty is the observation that approximately half of CF individuals are not aware of the possibility of developing CFRD as a complication of CF. ${ }^{97,99}$ To avoid patients feeling blind-sighted by the diagnosis of CFRD, CF caregivers might introduce the possibility of CFRD development as a common complication in $\mathrm{CF}$ at an earlier age. This can be achieved through educational materials, family/patient education events, and interaction with experts in CFRD.

The diagnosis of CFRD can be especially challenging in the pediatric population; CFRD is often diagnosed in the adolescent ages, a time during which children are learning to deal with the burdens of a chronic disease and exercising autonomy. The management of CFRD can significantly disrupt the lives of these individuals, more so during acute illnesses when CF disease treatment is already taxing. However, some individuals take on greater responsibility and ownership of diabetes and are able to incorporate CFRD into CF care. Managing CFRD can help encourage adolescents to participate more in routine $\mathrm{CF}$ care.

Treatment recommendations can be rejected or poorly followed by the patient, and therefore a close partnership between the CF care team including an endocrinologist and the patient must be developed to overcome individual barriers to CFRD care. Education regarding the etiology and implications of CFRD, insulin therapy, blood glucose monitoring, treatment of hypo- and hyperglycemia, the effects of food intake, stress, illness, and physical activity is crucial for successful partnership in the management of CFRD. Along with the pain of insulin injections, frequent blood glucose monitoring is cumbersome. Patients are most likely to avoid blood glucose checks, erroneously assuming (especially during illnesses) that an estimated insulin dose is sufficient. The importance of glucose monitoring needs to be repeatedly emphasized at CFRD visits. The most prevalent misconception regarding CFRD is that calories should be restricted. This is in conflict with $\mathrm{CF}$ care but reflects what is common knowledge regarding care of T2DM. Therefore, a nutritional plan should be discussed by the CFRD team and the patient educated regarding caloric intake with a focus on healthy calories.

\section{Future directions}

The mechanisms underlying insulin secretion defects, diabetes screening, and the role of earlier treatment on nutritional status, and pulmonary function are presently under further investigation.

Important areas where further study is crucial and would address management concerns include:

1. the mechanistic links between insulin secretion defects, glucose intolerance, and worse clinical outcomes are still vague. Hyperglycemia is postulated to increase infection susceptibility and to perpetuate the inflammatory cascade while insulin deficiency results in increased proteolysis and presumably loss of muscle mass.

2. Optimal screening practices remain a challenge, and the intervals at which screening should occur with respect to CF status, age, and previous glucose tolerance have yet to be assessed.

3. Earlier treatment of glucose abnormalities either in the IGT or indeterminate phase may have implications for progression of not only CF lung disease but insulin secretion abnormalities.

4. With the advent of ivacaftor therapy, assessment of insulin secretion and improvements in glucose tolerance related to ivacaftor are also exciting areas of research. As other disease modifying agents become available, their impact upon glucose intolerance and insulin secretion will need formal consideration.

\section{Conclusion}

Improved survival in $\mathrm{CF}$ has brought $\mathrm{CFRD}$ to the forefront of $\mathrm{CF}$ care. The $\mathrm{CF}$ community recognizes the negative effects of CFRD on pulmonary health and efforts are underway on both the research and clinical fronts to understand and manage CFRD. With further characterization of insulin defects and the mechanisms by which hyperglycemia adversely affects clinical status, improved screening practices, and novel therapeutics, the threat CFRD poses can be countered.

\section{Disclosure}

The authors do not have any conflicts of interest.

\section{References}

1. Moran A, Becker D, Casella SJ, et al. Epidemiology, pathophysiology, and prognostic implications of cystic fibrosis-related diabetes: a technical review. Diabetes Care. 2010;33(12):2677-2683.

2. Moran A, Dunitz J, Nathan B, Saeed A, Holme B, Thomas W. Cystic fibrosis-related diabetes: current trends in prevalence, incidence, and mortality. Diabetes Care. 2009;32(9):1626-1631.

3. Finkelstein SM, Wielinski CL, Elliott GR, et al. Diabetes mellitus associated with cystic fibrosis. J Pediatr. 1988;112(3):373-377. 
4. Lewis C, Blackman SM, Nelson A, et al. Diabetes-related mortality in adults with cystic fibrosis. Role of genotype and sex. Am J Respir Crit Care Med. 2015;191(2):194-200.

5. Sheikh S, Zemel BS, Stallings VA, Rubenstein RC, Kelly A. Body composition and pulmonary function in cystic fibrosis. Front Pediatr. 2014;2:33.

6. Kerem E, Viviani L, Zolin A, et al. Factors associated with FEV1 decline in cystic fibrosis: analysis of the ECFS patient registry. Eur Respir J. 2014;43(1):125-133.

7. Koch C, Rainisio M, Madessani U, et al. Presence of cystic fibrosisrelated diabetes mellitus is tightly linked to poor lung function in patients with cystic fibrosis: data from the European Epidemiologic Registry of Cystic Fibrosis. Pediatr Pulmonol. 2001;32(5):343-350.

8. Milla CE, Warwick WJ, Moran A. Trends in pulmonary function in patients with cystic fibrosis correlate with the degree of glucose intolerance at baseline. Am J Respir Crit Care Med. 2000;162(3 Pt 1): 891-895.

9. Lanng S, Thorsteinsson B, Nerup J, Koch C. Influence of the development of diabetes mellitus on clinical status in patients with cystic fibrosis. Eur J Pediatr. 1992;151(9):684-687.

10. Sequeiros IM, Jarad N. Factors associated with a shorter time until the next pulmonary exacerbation in adult patients with cystic fibrosis. Chron Respir Dis. 2012;9(1):9-16.

11. Bismuth E, Laborde K, Taupin P, et al. Glucose tolerance and insulin secretion, morbidity, and death in patients with cystic fibrosis. J Pediatr. 2008;152(4):540-545, 545.e1.

12. Bradbury RA, Shirkhedkar D, Glanville AR, Campbell LV. Prior diabetes mellitus is associated with increased morbidity in cystic fibrosis patients undergoing bilateral lung transplantation: an 'orphan' area? A retrospective case-control study. Intern Med J. 2009;39(6):384-388.

13. Brennan AL, Gyi KM, Wood DM, et al. Airway glucose concentrations and effect on growth of respiratory pathogens in cystic fibrosis. J Cyst Fibros. 2007;6(2):101-109.

14. Garnett JP, Gray MA, Tarran R, et al. Elevated paracellular glucose flux across cystic fibrosis airway epithelial monolayers is an important factor for Pseudomonas aeruginosa growth. PLoS One. 2013;8(10):e76283.

15. Van Sambeek L, Cowley ES, Newman DK, Kato R. Sputum glucose and glycemic control in cystic fibrosis-related diabetes: a cross-sectional study. PLoS One. 2015;10(3):e0119938.

16. Rolon MA, Benali K, Munck A, et al. Cystic fibrosis-related diabetes mellitus: clinical impact of prediabetes and effects of insulin therapy. Acta Paediatr. 2001;90(8):860-867.

17. Nousia-Arvanitakis S, Galli-Tsinopoulou A, Karamouzis M. Insulin improves clinical status of patients with cystic-fibrosis-related diabetes mellitus. Acta Paediatr. 2001;90(5):515-519.

18. Dobson L, Hattersley AT, Tiley S, Elworthy S, Oades PJ, Sheldon CD. Clinical improvement in cystic fibrosis with early insulin treatment Arch Dis Child. 2002;87(5):430-431.

19. Ripa P, Robertson I, Cowley D, Harris M, Masters IB, Cotterill AM. The relationship between insulin secretion, the insulin-like growth factor axis and growth in children with cystic fibrosis. Clin Endocrinol (Oxf). 2002;56(3):383-389.

20. Bizzarri C, Montemitro E, Pedicelli S, et al. Glucose tolerance affects pubertal growth and final height of children with cystic fibrosis. Pediatr Pulmonol. 2015;50(2):144-149.

21. Hameed S, Morton JR, Jaffe A, et al. Early glucose abnormalities in cystic fibrosis are preceded by poor weight gain. Diabetes Care 2010;33(2):221-226.

22. Bizzarri C, Lucidi V, Ciampalini P, Bella S, Russo B, Cappa M. Clinical effects of early treatment with insulin glargine in patients with cystic fibrosis and impaired glucose tolerance. J Endocrinol Invest. 2006; 29(3):RC1-RC4.

23. Hameed S, Morton JR, Field PI, et al. Once daily insulin detemir in cystic fibrosis with insulin deficiency. Arch Dis Child. 2012;97(5): 464-467.
24. Mozzillo E, Franzese A, Valerio G, et al. One-year glargine treatment can improve the course of lung disease in children and adolescents with cystic fibrosis and early glucose derangements. Pediatr Diabetes. 2009;10(3):162-167.

25. Schwarzenberg SJ, Thomas W, Olsen TW, et al. Microvascular complications in cystic fibrosis-related diabetes. Diabetes Care. 2007;30(5): 1056-1061.

26. van den Berg JM, Morton AM, Kok SW, Pijl H, Conway SP, Heijerman HG. Microvascular complications in patients with cystic fibrosis-related diabetes (CFRD). J Cyst Fibros. 2008;7(6):515-519.

27. Quon BS, Mayer-Hamblett N, Aitken ML, Smyth AR, Goss CH. Risk factors for chronic kidney disease in adults with cystic fibrosis. Am J Respir Crit Care Med. 2011;184(10):1147-1152.

28. American Diabetes Association. Diagnosis and classification of diabetes mellitus. Diabetes Care. 2013;36 Suppl 1:S67-S74.

29. Iannucci A, Mukai K, Johnson D, Burke B. Endocrine pancreas in cystic fibrosis: an immunohistochemical study. Hum Pathol. 1984;15(3): 278-284.

30. Abdul-Karim FW, Dahms BB, Velasco ME, Rodman HM. Islets of Langerhans in adolescents and adults with cystic fibrosis. A quantitative study. Arch Pathol Lab Med. 1986;110(7):602-606.

31. Soejima K, Landing BH. Pancreatic islets in older patients with cystic fibrosis with and without diabetes mellitus: morphometric and immunocytologic studies. Pediatr Pathol. 1986;6(1):25-46.

32. Adler AI, Shine BS, Chamnan P, Haworth CS, Bilton D. Genetic determinants and epidemiology of cystic fibrosis-related diabetes: results from a British cohort of children and adults. Diabetes Care. 2008;31(9):1789-1794.

33. Mohan K, Miller H, Dyce P, et al. Mechanisms of glucose intolerance in cystic fibrosis. Diabet Med. 2009;26(6):582-588.

34. No authors listed. Abstracts of the 27th Annual North American Cystic Fibrosis Conference. October 17-19, 2013. Salt Lake City, Utah, USA. Pediatr Pulmonol. 2013;48 Suppl 36:103-472.

35. Cucinotta D, Conti Nibali S, Arrigo T, et al. Beta cell function, peripheral sensitivity to insulin and islet cell autoimmunity in cystic fibrosis patients with normal glucose tolerance. Horm Res. 1990;34(1): 33-38

36. Moran A, Diem P, Klein DJ, Levitt MD, Robertson RP. Pancreatic endocrine function in cystic fibrosis. J Pediatr. 1991;118(5):715-723.

37. Cano Megias M, Gonzalez Albarran O, Guisado Vasco P, Lamas Ferreiro A, Maiz Carro L. Insulin resistance, beta-cell dysfunction and differences in curves of plasma glucose and insulin in the intermediate points of the standard glucose tolerance test in adults with cystic fibrosis. Endocrinol Nutr. 2015;62(2):91-99.

38. Rakotoambinina B, Delaisi B, Laborde $\mathrm{K}$, et al. Insulin responses to intravenous glucose and the hyperglycemic clamp in cystic fibrosis patients with different degrees of glucose tolerance. Pediatr Res. 1994; 36(5):667-671.

39. Lombardo F, De Luca F, Rosano M, et al. Natural history of glucose tolerance, beta-cell function and peripheral insulin sensitivity in cystic fibrosis patients with fasting euglycemia. Eur J Endocrinol. 2003; 149(1):53-59.

40. Cucinotta D, De Luca F, Gigante A, et al. No changes of insulin sensitivity in cystic fibrosis patients with different degrees of glucose tolerance: an epidemiological and longitudinal study. Eur J Endocrinol. 1994;130(3):253-258.

41. Knop FK, Vilsboll T, Larsen S, et al. Increased postprandial responses of GLP-1 and GIP in patients with chronic pancreatitis and steatorrhea following pancreatic enzyme substitution. Am J Physiol Endocrinol Metab. 2007;292(1):E324-E330.

42. Kelly A, Moran A. Update on cystic fibrosis-related diabetes. J Cyst Fibros. 2013;12(4):318-331.

43. Perano SJ, Couper JJ, Horowitz M, et al. Pancreatic enzyme supplementation improves the incretin hormone response and attenuates postprandial glycemia in adolescents with cystic fibrosis: a randomized crossover trial. J Clin Endocrinol Metab. 2014;99(7): 2486-2493. 
44. O'Riordan SM, Robinson PD, Donaghue KC, Moran A; ISPAD Clinical Practice Consensus. Management of cystic fibrosis-related diabetes. Pediatr Diabetes. 2008;9(4 Pt 1):338-344.

45. Yung B, Noormohamed FH, Kemp M, Hooper J, Lant AF, Hodson ME. Cystic fibrosis-related diabetes: the role of peripheral insulin resistance and beta-cell dysfunction. Diabet Med. 2002;19(3):221-226.

46. Smerieri A, Montanini L, Maiuri L, Bernasconi S, Street ME. FOXO1 content is reduced in cystic fibrosis and increases with IGF-I treatment. Int J Mol Sci. 2014;15(10):18000-18022.

47. Moran A, Basu R, Milla C, Jensen MD. Insulin regulation of free fatty acid kinetics in adult cystic fibrosis patients with impaired glucose tolerance. Metabolism. 2004;53(11):1467-1472.

48. Hardin DS, LeBlanc A, Lukenbaugh S, Para L, Seilheimer DK. Proteolysis associated with insulin resistance in cystic fibrosis. Pediatrics. 1998;101(3 Pt 1):433-437.

49. Hanna RM, Weiner DJ. Overweight and obesity in patients with cystic fibrosis: a center-based analysis. Pediatr Pulmonol. 2015;50(1):35-41.

50. Donath MY, Halban PA. Decreased beta-cell mass in diabetes: significance, mechanisms and therapeutic implications. Diabetologia. 2004;47(3):581-589.

51. Butler AE, Janson J, Bonner-Weir S, Ritzel R, Rizza RA, Butler PC. Beta-cell deficit and increased beta-cell apoptosis in humans with type 2 diabetes. Diabetes. 2003;52(1):102-110.

52. Hotamisligil GS. Inflammation and metabolic disorders. Nature. 2006;444(7121):860-867.

53. Luciani A, Villella VR, Esposito S, et al. Cystic fibrosis: a disorder with defective autophagy. Autophagy. 2011;7(1):104-106.

54. Maiese K. New Insights for Oxidative Stress and Diabetes Mellitus. Oxid Med Cell Longev. 2015;2015:875961.

55. Blackman SM, Hsu S, Ritter SE, et al. A susceptibility gene for type 2 diabetes confers substantial risk for diabetes complicating cystic fibrosis. Diabetologia. 2009;52(9):1858-1865.

56. Blackman SM, Commander CW, Watson C, et al. Genetic modifiers of cystic fibrosis-related diabetes. Diabetes. 2013;62(10):3627-3635.

57. Park JH, Stoffers DA, Nicholls RD, Simmons RA. Development of type 2 diabetes following intrauterine growth retardation in rats is associated with progressive epigenetic silencing of $\mathrm{Pdx} 1$. J Clin Invest. 2008;118(6):2316-2324.

58. Sandovici I, Smith NH, Nitert MD, et al. Maternal diet and aging alter the epigenetic control of a promoter-enhancer interaction at the Hnf4a gene in rat pancreatic islets. Proc Natl Acad Sci U S A. 2011;108(13): 5449-5454.

59. Dayeh T, Volkov P, Salo S, et al. Genome-wide DNA methylation analysis of human pancreatic islets from type 2 diabetic and non-diabetic donors identifies candidate genes that influence insulin secretion. PLoS Genet. 2014;10(3):e1004160.

60. Boom A, Lybaert P, Pollet JF, et al. Expression and localization of cystic fibrosis transmembrane conductance regulator in the rat endocrine pancreas. Endocrine. 2007;32(2):197-205.

61. Olivier AK, Yi Y, Sun X, et al. Abnormal endocrine pancreas function at birth in cystic fibrosis ferrets. J Clin Invest. 2012;122(10):3755-3768.

62. Guo JH, Chen H, Ruan YC, et al. Glucose-induced electrical activities and insulin secretion in pancreatic islet beta-cells are modulated by CFTR. Nat Commun. 2014;5:4420.

63. Uc A, Olivier AK, Griffin MA, et al. Glycaemic regulation and insulin secretion are abnormal in cystic fibrosis pigs despite sparing of islet cell mass. Clin Sci (Lond). 2015;128(2):131-142.

64. Bellin MD, Laguna T, Leschyshyn J, et al. Insulin secretion improves in cystic fibrosis following ivacaftor correction of CFTR: a small pilot study. Pediatr Diabetes. 2013;14(6):417-421.

65. Hayes D Jr, McCoy KS, Sheikh SI. Resolution of cystic fibrosisrelated diabetes with ivacaftor therapy. Am J Respir Crit Care Med. 2014;190(5):590-591.

66. Moran A, Brunzell C, Cohen RC, et al. Clinical care guidelines for cystic fibrosis-related diabetes: a position statement of the American Diabetes Association and a clinical practice guideline of the Cystic Fibrosis Foundation, endorsed by the Pediatric Endocrine Society. Diabetes Care. 2010;33(12):2697-2708.
67. Moran A, Pekow P, Grover P, et al. Insulin therapy to improve BMI in cystic fibrosis-related diabetes without fasting hyperglycemia: results of the cystic fibrosis related diabetes therapy trial. Diabetes Care. 2009; 32(10): $1783-1788$.

68. Scheuing N, Holl RW, Dockter G, et al. High variability in oral glucose tolerance among 1,128 patients with cystic fibrosis: a multicenter screening study. PLoS One. 2014;9(11):e112578.

69. Sterescu AE, Rhodes B, Jackson R, et al. Natural history of glucose intolerance in patients with cystic fibrosis: ten-year prospective observation program. J Pediatr. 2010;156(4):613-617.

70. Lanng S, Hansen A, Thorsteinsson B, Nerup J, Koch C. Glucose tolerance in patients with cystic fibrosis: five year prospective study. $B M J$. 1995;311(7006):655-659.

71. Dobson L, Sheldon CD, Hattersley AT. Conventional measures underestimate glycaemia in cystic fibrosis patients. Diabet Med. 2004;21(7): 691-696.

72. Street ME, Spaggiari C, Ziveri MA, et al. Insulin production and resistance in cystic fibrosis: effect of age, disease activity, and genotype. $J$ Endocrinol Invest. 2012;35(3):246-253.

73. Cucinotta D, De Luca F, Scoglio R, et al. Factors affecting diabetes mellitus onset in cystic fibrosis: evidence from a 10-year follow-up study. Acta Paediatr. 1999;88(4):389-393.

74. Marshall BC, Butler SM, Stoddard M, Moran AM, Liou TG, Morgan WJ. Epidemiology of cystic fibrosis-related diabetes. J Pediatr. 2005;146(5):681-687.

75. Garagorri JM, Rodriguez G, Ros L, Sanchez A. Early detection of impaired glucose tolerance in patients with cystic fibrosis and predisposition factors. J Pediatr Endocrinol Metab. 2001;14(1): 53-60.

76. Patient Registry. Annual data report to the Center directors. 2013. Cystic Fibrosis Foundation. Available from: https://www.cff.org/2013_CFF_ Annual_Data_Report_to_the_Center_Directors.pdf. Accessed May 14, 2015.

77. Lee KM, Miller RJ, Rosenberg FM, Kreisman SH. Evaluation of glucose tolerance in cystic fibrosis: comparison of 50-g and 75 -g tests. $J$ Cyst Fibros. 2007;6(4):274-276.

78. Dobson L, Sheldon CD, Hattersley AT. Validation of interstitial fluid continuous glucose monitoring in cystic fibrosis. Diabetes Care. 2003;26(6):1940-1941.

79. Franzese A, Valerio G, Buono P, et al. Continuous glucose monitoring system in the screening of early glucose derangements in children and adolescents with cystic fibrosis. J Pediatr Endocrinol Metab. 2008;21(2):109-116.

80. Moreau F, Weiller MA, Rosner V, et al. Continuous glucose monitoring in cystic fibrosis patients according to the glucose tolerance. Horm Metab Res. 2008;40(7):502-506.

81. Schiaffini R, Brufani C, Russo B, et al. Abnormal glucose tolerance in children with cystic fibrosis: the predictive role of continuous glucose monitoring system. Eur J Endocrinol. 2010;162(4):705-710.

82. O'Riordan SM, Hindmarsh P, Hill NR, et al. Validation of continuous glucose monitoring in children and adolescents with cystic fibrosis: a prospective cohort study. Diabetes Care. 2009;32(6):1020-1022.

83. Godbout A, Hammana I, Potvin S, et al. No relationship between mean plasma glucose and glycated haemoglobin in patients with cystic fibrosis-related diabetes. Diabetes Metab. 2008;34(6 Pt 1):568-573.

84. Solomon MP, Wilson DC, Corey M, et al. Glucose intolerance in children with cystic fibrosis. J Pediatr. 2003;142(2):128-132.

85. Burgess JC, Bridges N, Banya $\mathrm{W}$, et al. $\mathrm{HbA}_{\mathrm{cc}}$ as a screening tool for cystic fibrosis related diabetes. J Cyst Fibros. Epub April 10, 2015.

86. Moran A. Diagnosis, screening, and management of cystic fibrosisrelated diabetes. Curr Diab Rep. 2002;2(2):111-115.

87. Tofe S, Moreno JC, Maiz L, Alonso M, Escobar H, Barrio R. Insulinsecretion abnormalities and clinical deterioration related to impaired glucose tolerance in cystic fibrosis. Eur J Endocrinol. 2005;152(2): 241-247.

88. Minicucci L, Haupt M, Casciaro R, et al. Slow-release insulin in cystic fibrosis patients with glucose intolerance: a randomized clinical trial. Pediatr Diabetes. 2012;13(2):197-202. 
89. Mohan K, Israel KL, Miller H, Grainger R, Ledson MJ, Walshaw MJ. Long-term effect of insulin treatment in cystic fibrosis-related diabetes. Respiration. 2008;76(2):181-186.

90. Lanng S, Thorsteinsson B, Nerup J, Koch C. Diabetes mellitus in cystic fibrosis: effect of insulin therapy on lung function and infections. Acta Paediatr. 1994;83(8):849-853.

91. Onady GM, Stolfi A. Insulin and oral agents for managing cystic fibrosisrelated diabetes. Cochrane Database Syst Rev. 2013;7:CD004730.

92. Spijker HS, Wolffenbuttel BH, van der Bij W, Engelse MA, Rabelink TJ, de Koning EJ. Islet-after-lung transplantation in a patient with cystic fibrosis-related diabetes. Diabetes Care. 2014;37(7):e159-e160.

93. Trujillo JM, Nuffer W, Ellis SL. GLP-1 receptor agonists: a review of head-to-head clinical studies. Ther Adv Endocrinol Metab. 2015;6(1): 19-28.

94. Horton ES, Silberman C, Davis KL, Berria R. Weight loss, glycemic control, and changes in cardiovascular biomarkers in patients with type 2 diabetes receiving incretin therapies or insulin in a large cohort database. Diabetes Care. 2010;33(8):1759-1765.
95. Rickels MR, Bellin M, Toledo FG, et al. Detection, evaluation and treatment of diabetes mellitus in chronic pancreatitis: recommendations from PancreasFest 2012. Pancreatology. 2013;13(4):336-342.

96. Kawalec P, Mikrut A, Lopuch S. The safety of dipeptidyl peptidase-4 (DPP-4) inhibitors or sodium-glucose cotransporter 2 (SGLT-2) inhibitors added to metformin background therapy in patients with type 2 diabetes mellitus: a systematic review and meta-analysis. Diabetes Metab Res Rev. 2014;30(4):269-283.

97. Clemmons DR. Metabolic actions of insulin-like growth factor-I in normal physiology and diabetes. Endocrinol Metab Clin North Am. 2012;41(2):425-443.

98. Ramsey BW, Davies J, McElvaney NG, et al. A CFTR potentiator in patients with cystic fibrosis and the G551D mutation. $N$ Engl J Med. 2011;365(18):1663-1672.

99. Millington K, Miller V, Rubenstein RC, Kelly A. Patient and parent perceptions of the diagnosis and management of cystic fibrosis-related diabetes. Journal of Clinical and Translational Endocrinology. 2014; 1(3):100-107

\section{Publish your work in this journal}

Research and Reports in Endocrine Disorders is an international, peerreviewed, open access journal publishing original research, reports, reviews and commentaries on all areas of endocrinology, endocrine disorders and therapeutic interventions. The manuscript management system is completely online and includes a very quick and fair

\section{Dovepress}

peer-review system. Visit http://www.dovepress.com/testimonials.php to read real quotes from published authors. 